\title{
Distúrbio alimentar, compulsivo e afetivo: uma revisão bibliográfica acerca da associação
}

\section{Eating, compulsive and affective disorder: a bibliographic review about the association}

Ivan Lucas Picone Borges dos Anjos ${ }^{\dagger}$, Lara Costa Martins ${ }^{\ddagger}$, Sara Cristine Marques dos Santos ${ }^{\dagger}$, Ivana Picone Borges de Aragão ${ }^{\S}$

Como citar esse artigo. Dos Anjos,

I. L. P. B.; Martins, L. C.; Dos

Santos, S. C. M; De Aragão, I. P.

B. Distúrbio alimentar, compulsivo

e afetivo: uma revisão bibliográfica

acerca da associação. Revista de

Saúde $2020 \mathrm{Jul} / \mathrm{Dez} ; 11$ (2): 60 - 64

\section{Resumo}

Os distúrbios alimentares se enquadram em um grupo de condições clínicas, entre elas, a compulsão alimentar, a bulimia e a anorexia. Já, entre os distúrbios compulsivos, o dimorfismo corporal configura uma de suas apresentações. No âmbito dos distúrbios afetivos, são identificados a ansiedade e a depressão. Do ponto de vista epidemiológico, os distúrbios alimentares vêm ocorrendo com grande frequência, havendo aumento do interesse pelo tema, o que contribuiu para as crescentes pesquisas, na tentativa de se estabelecerem os critérios diagnósticos dos transtornos alimentares, nas últimas décadas. Frequentemente, transtornos alimentares, afetivos e de dismorfismo corporal ocorrem de forma concomitante e complementar, sugerindo a intercausalidade dos transtornos. Foi realizada revisão bibliográfica, utilizando 27 artigos selecionados, no período entre 1984 e 2019, nas seguintes bases de dados científicos: PubMed (26) e SciELO (01). Estudos são necessários para comprovar a associação entre transtornos alimentares, afetivos e de dismorfismo corporal, favorecendo a prevenção primária e a otimização do tratamento, assim como a conscientização e a educação dos pacientes e familiares para a identificação precoce e apoio ao tratamento.

Palavras-chave: Imagem Corporal, Comportamento alimentar, Transtorno de Humor, Depressão, Ansiedade.

\begin{abstract}
Eating disorders fall within a group of clinical conditions, including binge eating, bulimia and anorexia. Among compulsive disorders, body dimorphism is one of his presentations. In the context of affective disorders, anxiety and depression are identified. From the epidemiological point of view, eating disorders have been occurring with great frequency, with an increase in interest in the theme, which has contributed to the increasing research, in an attempt to establish the diagnostic criteria of eating disorders in recent decades. Often, eating disorders, affective disorders and body dysmorphism occur concomitantly and complementarily, suggesting the intercausality of the disorders. A literature review was carried out, using 27 selected articles, between 1984 and 2019, in the following scientific databases: PubMed (26) and SciELO (01). Studies are necessary to prove the association between eating disorders, affective disorders and body dysmorphism, favoring primary prevention and treatment optimization, as well as the awareness and education of patients and family members for early identification and treatment support.
\end{abstract}

Keywords: Body Image, Feeding Behavior, Mood disorders, Depression, Anxiety.

\section{Introdução}

A sociedade atual se encontra cada vez mais influenciada pela busca de um "corpo perfeito", determinando padrões de estética restritos, que são almejados pela população. Frequentemente, esse processo resulta em alterações da percepção corporal por parte dos grupos sociais, produzindo um impacto negativo na autoestima dos jovens, assim como incapacidade de perceber sua aparência de forma positiva.

Parcela importante de jovens, influenciados por estímulos sociais e propensão genética, está exposta a desenvolver distúrbios alimentares, como anorexia ou bulimia, além de distúrbios afetivos, incluindo ansiedade ou depressão e transtornos obsessivos-compulsivos, como dismorfismo corporal.

Os transtornos alimentares afetam, em geral, adolescentes e jovens em idade produtiva do sexo feminino, culminando em prejuízos a nível pessoal,

${ }^{\dagger}$ Discente PIBIC do curso de Medicina da Universidade de Vassouras, Vassouras, Rio de Janeiro, Brasil

${ }^{\ddagger}$ Discente do curso de Medicina da Universidade de Vassouras, Vassouras, Rio de Janeiro, Brasil

${ }^{\S}$ Docente do curso de Medicina da Universidade de Vassouras, Vassouras, Rio de Janeiro, Brasil

* Email de correspondência: ilpba@hotmail.com
} 
com aumento da morbidade e mortalidade, e a nível social, devido ao absenteísmo resultante. É necessário identificar a prevalência de tais transtornos, uma vez que amaçam a saúde e a qualidade de vida do paciente e sua família.

O objetivo desse estudo foi identificar a prevalência e a correlação entre depressão, ansiedade e distúrbios relacionados com a autoimagem e distúrbios alimentares, podendo, assim, incentivar o rastreio dessas doenças, despertar a auto-percepção para um distúrbio alimentar e psiquiátrico, além de conscientizar a população acometida sobre a busca e opções de tratamento

\section{Métodos}

A presente revisão bibliográfica foi elaborada a partir de uma pesquisa, na qual foram selecionados 27 artigos, publicados no período entre 1984 e 2019, na base de dados científicos PubMed e SiELO.

$\mathrm{Na}$ base de dados PubMed foram revisados 26 artigos, dos quais: 05 pelo descritor em ciências da saúde "binge-eating"; 03 pelo descritor "anxiety"; 05 pelo descritor "depression"; 06 pelos descritores "body dysmorphic disorder"; 04 pelo descritor "bulimia"; 03 pelo descritor "anorexia nervosa" e 04 pelo descritor "affective disorder". Nas SciELO foi revisado 01 artigo com o descritor "comportamento alimentar"

\section{Revisão Bibliográfica}

Do ponto de vista epidemiológico, os distúrbios alimentares vêm recebendo grande atenção, havendo aumento do interesse pelo tema, o que contribuiu para as crescentes pesquisas, na tentativa de se estabelecerem os critérios diagnósticos dos transtornos alimentares, nas últimas décadas.

$\mathrm{O}$ primeiro distúrbio alimentar descrito foi a anorexia nervosa (AN) no século XIX e, igualmente, a pioneira a ser adequadamente classificada e ter critérios operacionais reconhecidos, já na década de 1970, sendo a bulimia nervosa (BN) em 1979'1.

Atualmente, vem sendo evidenciada forte relação entre os distúrbios alimentares, distúrbios compulsivos e distúrbios afetivos, seja essa relação causal ou complementar. Os distúrbios alimentares analisados serão a compulsão alimentar, a bulimia e a anorexia. Os distúrbios compulsivos analisado será o dimorfismo corporal. Os distúrbios afetivos analisados serão a ansiedade e a depressão.

Compulsão alimentar é a necessidade de comer, mesmo sem fome. Foi categorizada como um transtorno obsessivo, com alteração do sistema recompensa neurológico e vias de atenção relacionada à comida. Acredita-se que essa alteração seja originada de uma má adaptação do circuito córtico estriatal que regula impulsos, motivações e sistema recompensa ${ }^{2}$. O transtorno pode estar relacionado com distúrbios metabólicos, como diabetes mellitus tipo 2 e síndrome metabólica, tendo em vista o ganho ponderal, além de distúrbios psiquiátricos, como depressão e ansiedade ${ }^{3,4}$.

Estudo com pacientes obesos revelou forte relação positiva e complementar entre compulsão alimentar, vício em comida e transtornos depressivos. A presença de qualquer uma dessas comorbidades foi um fator de risco para instalação das outras ${ }^{4}$. Em mulheres, um estudo relacionou, positivamente, o tempo assistindo televisão e "reality shows", com o desenvolvimento de transtornos alimentares, depressão e ansiedade. Isso costuma ser identificado, pelo desejo do paciente, em se assemelhar com o personagem de televisão ${ }^{5}$.

Pacientes com compulsão alimentar podem ser diferenciados por sua distorção de autoimagem, sendo observada superestimação do peso e formato corporais, associados com maior insatisfação pessoal ${ }^{6,7}$. Estudo revelou que a terapia cognitivo comportamental é essencial para pacientes obesos com compulsão alimentar, seja realizando dietas restritivas ou dietas genéricas para perda de peso. Em ambos os casos, foi verificado uma redução de $5 \%$ do peso corporal, em $30 \%$ da população estudada ${ }^{8}$.

Acredita-se que distúrbios alimentares podem ser causados ou agravados por dietas restritivas ou períodos mais longos, sem ingerir alimentos. Porém, essa ideia foi contraposta em outro estudo, o qual revelou que indivíduos com compulsão alimentar e/ou bulimia, e sem qualquer transtorno, comiam o mesmo montante quando separados em grupos, tanto com alimentação a cada 01 hora, como a cada 06 horas. Ou seja, sem diferença significativa de ingestão calórica entre os grupos?.

A depressão e a ansiedade são a forma mais comum de distúrbios afetivos relacionados com a anorexia nervosa. Essa associação é mantida em todas as fases do tratamento, podendo serem agravados os sintomas de depressão, de acordo com o ganho de peso no decorrer do tratamento ${ }^{6}$. A insatisfação corporal, definida pela diferença entre uma silhueta desejada por um paciente, e sua silhueta atual, é mais comum em meninas do que em meninos e aumenta com a progressão da idade ${ }^{10}$.

A autoimagem corporal, que é definida como a construção multidimensional que engloba autopercepção e atitudes em relação à aparência física, afeta as emoções, pensamentos e comportamento no cotidiano, influenciando, principalmente, os relacionamentos interpessoais ${ }^{11}$. A autoimagem costuma ser influenciada por parceiros românticos, afetando mais mulheres do que homens, devido à maior pressão social exercida 
sobre elas ${ }^{10}$. A vergonha é um fator crucial que deve ser valorizado e contrabalanceado com incentivo positivo, uma vez que indivíduos, com alteração da autoimagem, alegam saber que outras pessoas não notam os defeitos que eles colocam sobre $\mathrm{si}^{11}$.

Indivíduos que possuem transtorno dismórfico corporal, podem apresentar delírios em relação à sua aparência e composição corporal, estando relacionado com a possibilidade de suicídio e automutilação, em proporção direta de sua intensidade ${ }^{11}$. Esses pacientes, frequentemente, se descrevem como feios, deformados, anormais e desfigurados, sendo progressivamente menos capazes de escutar uma opinião alheia, quanto maior for o delírio, enquanto um paciente, não delirante, é capaz de acreditar na informação de outra pessoa ${ }^{11}$.

$\mathrm{O}$ aumento do índice de massa corporal foi relacionado com a depressão, uma vez que a depressão não só aumenta a percepção da dimensão corporal, mas também a insatisfação corporal ${ }^{12}$. Contrariamente, um estudo conduzido em Nevada verificou que entre 1.162 adolescentes, as meninas tinham percepção de si mesmas, como sendo mais gordas do que realmente eram, enquanto os meninos, como sendo mais magros ${ }^{13}$.

A estigmatização da obesidade infantil é capaz de provocar aumento na incidência de depressão, insatisfação corporal, e aumento do incide de massa corporal. A redução do estigma pode promover melhor saúde mental em crianças e à longo prazo quando adultos $^{14}$.

O vício em comida já foi tanto relacionado, negativamente, com depressão e imagem corporal, como relacionado positivamente, com escores de depressão. A relação positiva entre o vício em comida e o índice de massa corporal também foi identificada no mesmo estudo $^{15}$

O transtorno de dismorfismo corporal é mais presente em mulheres, indivíduos que procuraram tratamentos estéticos e indivíduos que são sedentários e não praticam atividade física ${ }^{16,17}$. A carga de trabalho, renda e idade se correlacionaram, negativamente, com a ansiedade social, sendo essa relação positiva quando relacionado ao índice de massa corporal. Esses resultados revelam que a ansiedade social e o transtorno dismórfico corporal podem ser influenciados de formas diferentes ${ }^{16}$.

Em indivíduos do sexo masculino foi observada visão distorcida da muscularidade do corpo, como possuindo menos massa muscular do que realmente apresentam ${ }^{20}$. Indivíduos com transtorno dismórfico corporal costumam dispensar horas do dia à atenção de sua aparência, e nas formas de melhorá-la, o que pode estar relacionado à perda de qualidade de vida ${ }^{18}$.

Estudos sugerem que a anorexia nervosa e o transtorno dismórfico corporal compartilham muitas semelhanças, como o grau de insatisfação com o próprio corpo. Enquanto a anorexia nervosa está mais relacionada com o formato do corpo e o peso, o transtorno dismórfico corporal parece se preocupar com outros aspectos, como a face ${ }^{19}$.

Pacientes que possuem transtorno dismórfico corporal comumente apresentaram, no passado, o uso de serviços psiquiátricos, ansiedade, depressão, transtornos obsessivos-compulsivos, e transtornos alimentares ${ }^{20}$. O transtorno de dismorfismo corporal é caracterizado pela preocupação com defeitos estéticos inexistentes. Normalmente, se inicia na infância ou adolescência. É acompanhado por comportamentos repetitivos e muito tempo gasto em frente ao espelho, podendo estar relacionado à interferência com a vida cotidiana, social e o trabalho ${ }^{21}$.

Segundo estudo, quando anorexia e/ou bulimia estão associadas com depressão, habitualmente, a depressão é secundária aos outros dois transtornos ${ }^{22}$. Outro estudo apontou que a bulimia e/ou anorexia nervosa, quando associada(s) à depressão, pode se iniciar antes, durante ou depois da instalação dos distúrbios alimentáres ${ }^{23}$.

Os transtornos alimentares começam, primariamente, na adolescência, apesar de não se saber com exatidão seu mecanismo fisiopatológico, porém sendo estabelecida a existência de forte influência do meio social e predisposição ao comportamento obsessivo-compulsivo ${ }^{24}$.

As características costumam ser compulsão por contagem de caloria, comportamento ritualístico, envolvendo alimentos, uso de laxativos, provocação de vômitos, assim como, outras características obsessivas compulsivas $^{24}$. São manifestações obsessivas compulsivas a repetição, o comportamento ritualístico, a limpeza excessiva, a ordem excessiva, o perfeccionismo, a rigidez, a avareza, a falta de escrúpulos e a forte autocritica ${ }^{24}$

Existe um componente disfórico nos pacientes com transtornos alimentares e, também, uma resposta não esperada ao tratamento com antidepressivos; esses fatores fazem pensar que exista um transtorno afetivo de base ${ }^{24}$.

Indivíduos com distúrbios alimentares costumam ser discretos quanto à sua exposição e não são cientes de sua condição ${ }^{24}$. Pacientes com bulimia e abuso de substâncias, como diuréticos ou laxativos, também costumam apresentar problemas financeiros, problemas relacionados ao trabalho ou convívio social e ao consumo excessivo de álcool, estando expostos a maior índice de suicídio ${ }^{25}$.Aqueles com transtorno afetivo, associado aos distúrbios alimentares, costumam buscar mais tratamento que os pacientes apenas com bulimia, que utilizam ou não substâncias laxativas ${ }^{25}$. Crianças, com distúrbios nutricionais e transtornos alimentares, demonstraram dificuldade na regulação de suas próprias emoções como impulsividade ou depressão/ retraimento ${ }^{26}$. 
Nos portadores de transtorno afetivo maior foram identificados como portadores de uma tríade de sintomas de transtornos alimentares: perturbação na consciência interoceptiva, sensação de ineficácia e tendência à bulimia. A sensação de ineficácia aparenta ser secundária à depressão maior, enquanto a bulimia é um fator independente que pode surgir devido à pressão familiar ou social ${ }^{27}$.

Os casos de bulimia com depressão maior possuem mais dificuldade de responder ao tratamento do que aqueles que possuem apenas bulimia ${ }^{27}$.

\section{Conclusão}

Tanto os distúrbios afetivos, quanto alimentares e compulsivos necessitam de uma predisposição genética para ocorrer. Entretanto, a exposição a fatores sociais estressantes, como padrões de beleza divulgados pela mídia e opiniões de terceiros, pode atuar como deflagradores ao desenvolvimento de baixa autoestima, que pode estar relacionada negativamente à futura alteração da autoimagem.

Os distúrbios alimentares, como a bulimia e a anorexia, são motivados pela busca de um ideal físico imposto pelo próprio paciente, enquanto os transtornos afetivos, como a depressão e a ansiedade, funcionam como uma resposta à falha na obtenção, aos olhos do mesmo, dos resultados almejados.

Frequentemente, os transtornos avaliados no texto são encontrados concomitantemente e de forma complementar, sugerindo a associação e, possível, intercausalidade dos transtornos afetivos, ansiedade e depressão, transtorno dismórfico corporal e dos transtornos alimentares, bulimia, anorexia nervosa e compulsão alimentar.

Mais estudos são necessários para comprovar, além da associação, a relação de causalidade entre eles, favorecendo a prevenção primária e a otimização do tratamento, assim como a conscientização e a educação dos pacientes e familiares para sua identificação precoce e apoio ao tratamento.

\section{Referências}

1. Freitas C, Almonfrey F, Sepulvida M, et al. Terapia anticoagulante 1 . Cordás TA. Transtornos alimentares: classificação e diagnóstico. Rev Psiq Clin 2004;31(4):154-157

2. Kessler RM, Hutson PH, Herman BK, Potenza MN. The neurobiological basis of binge-eating disorder. Neurosci Biobehav Rev. 2016;63:223-238.

3. Kornstein SG, Kunovac JL, Herman BK, Culpepper L. Recognizing Binge-Eating Disorder in the Clinical Setting: A Review of the Literature. Prim Care Companion CNS Disord. 2016;18(3).

4. Imperatori $\mathrm{C}$, Innamorati $\mathrm{M}$, Contardi A, Continisio M, Tamburello S, Lamis DA, et al. The association among food addiction, binge eating severity and psychopathology in obese and overweight patients attending low-energydiet therapy. Comprehensive Psychiatry. 2014;55(6):1358-1362.
5. Barcaccia B, Balestrini V, Saliani AM, Baiocco R, Mancini F, Schneider BH. Dysfunctional eating behaviors, anxiety, and depression in Italian boys and girls: the role of mass media. 2018;40(1):72-77.

6. Junne F, Zipfel S, Wild B, Martus P, Giel K, Resmark G, et al. The relationship of body image with symptoms of depression and anxiety in patients with anorexia nervosa during outpatient psychotherapy: Results of the ANTOP study. Psychotherapy (Chic). 2016;53(2):141-51.

7. Yiu A1, Murray SM1, Arlt JM1, Eneva KT1, Chen EY2. The importance of body image concerns in overweight and normal-weight individuals with binge eating disorder. Body Image. 2017;22:6-12 7

8. Masheb RM, Griloa CM, Rollsb BJ. A randomized controlled trial for obesity and binge eating disorder: Low-energy-density dietary counseling and cognitive-behavioral therapy. Behaviour Research and Therapy. 2011;49(12):821-829.

9. Telch CF, Agras WS. The Effects of Short-Term Food Deprivation on Caloric Intake in Eating-Disordered Subjects. Appetite. 1996;26(3):221234.

10. Flores PJ, Cruz AJ, Gascón MB. Body-image dissatisfaction in children and adolescents: a systematic review. Nutr Hosp. 2017;34(2):479-489.

11. Tomas-Aragones L, Marron SE. Body Image and Body Dysmorphic Concerns. Acta Derm Venereol. 2016;96(217):47-50.

12. Paans NPG, Bot M, Brouwer IA, Visser M, Penninx BWJH. Contributions of depression and body mass index to body image. Journal of Psychiatric Research. 2018;103:18-25

13. Ruiz MNS, Fernández BM, Ontoso IA, Guillén-Grima F, Monzó IS, Armayor NC, et al. Analysis of body image perception of university students in Navarra. Nutr Hosp. 2015;31(5):2269-2275.

14. Stevens SD, Herbozo S, Morrell HE, Schaefer LM, Thompson JK. Adult and childhood weight influence body image and depression through weight stigmatization. J Health Psychol. 2017;22(8):1084-1093.

15. Şanlier N, Türközü D, Toka O. Body Image, Food Addiction, Depression, and Body Mass Index in University Students. Ecol Food Nutr. 2016;55(6):491-507.

16. Soler T, Novaes J, Fernandes M. Influencing Factors of Social Anxiety Disorder and Body Dysmorphic Disorder in a Nonclinical Brazilian Population. Psychol Rep. 2018.

17. More KR, Phillips LA, Colman MHE. Evaluating the potential roles of body dissatisfaction in exercise avoidance. Body Image. 2019;28:110114.

18. Perkins A. Body dysmorphic disorder: The drive for perfection. Nursing. 2019 Mar;49(3):28-33.

19. Phillipou A, Castle DJ, Rossell SL. Direct comparisons of anorexia nervosa and body dysmorphic disorder: A systematic review. Psychiatry Res. 2019;274:129-137.

20. Schneider SC, Mond J, Turner CM, Hudson JL. Sex Differences in the Presentation of Body Dysmorphic Disorder in a Community Sample of Adolescents. J Clin Child Adolesc Psychol. 2019;48(3):516-528.

21. Hardardottir H, Hauksdottir A, Bjornsson AS. Body dysmorphic disorder: Symptoms, prevalence, assessment and treatment Laeknabladid. 2019;105(3):125-131.

22. Laessle RG, Kittl S, Fichter MM, Wittchen HU, Pirke KM. Major affective disorder in anorexia nervosa and bulimia. A descriptive diagnostic study. Br J Psychiatry. 1987;151:785-9.

23. Katz JL. Eating disorder and affective disorder: relatives or merely chance acquaintances? Compr Psychiatry. 1987;28(3):220-8.

24. Rothenberg A. Adolescence and eating disorder: the obsessive-compulsive syndrome. Psychiatr Clin North Am. 1990;13(3):469-88.

25. Hatsukami D, Mitchell JE, Eckert ED, Pyle R. Characteristics of patients with bulimia only, bulimia with affective disorder, and bulimia with substance abuse problems. Addict Behav 1986;11(4):399-406.

26. Cimbolli P, Quiñones Á, Ugarte C, De Pascale A. Pilot study on nutritional and eating disorders in children and mood disorders: comorbidity 
or prodromal traits? Rev Psichiatr. 2017;52(1):32-39.

27. Wold PN. Eating disorder symptoms in affective disorder. J Psychiatry

Neurosci. 1991;16(4):204-8. 